ARTíCULO

\title{
Análisis bioinformático del sistema flagelar de la alphaproteobacteria tipo rickettsia Candidatus Hepatobacter penaei
}

Bioinformatic analysis of the flagellar system of the rickettsia-like alphaproteobacteria Candidatus Hepatobacter penaei

\author{
Juan Manuel Leyva ${ }^{1}$, Marcel Martínez-Porchas ${ }^{1}$, Francisco \\ Vargas-Albores ${ }^{1}$, Jorge Hernández-López ${ }^{2}$, Teresa Gollas-Galván ${ }^{1 *}$
}

\author{
${ }^{1}$ Centro de Investigación en Alimentación y Desarrollo, A.C, Carretera a La Victoria km. 0.6, C.P. 83304, Hermosillo, Sonora, México. \\ *tgollas@ciad.mx \\ ${ }^{2}$ Centro de Investigaciones Biológicas del Noroeste, S.C., Hermosa 101, Col. Los Ángeles, CP. 83106, Hermosillo, Sonora, México
}

\begin{abstract}
The presence of flagellar systems is common in several free-living bacteria; however, these have been considered absent in bacteria belonging to the Rickettsiales order. Candidatus Hepatobacter penaei is a marine Rickettsial bacterium described as a motile microorganism propelled by a flagellar system that facilitates infection. This bacterium represents a major risk of infection in shrimp farms. Therefore, the objective of this study was to determine the presence of genes encoding the flagellar system proteins of the bacterium Candidatus Hepatobacter penaei. The genomic sequence of the GenBank database with accession number NZ_JQAJ00000000.1, was analyzed. After genes were identified, amino acid sequences were deduced. The amino acid sequences of the flagellar system were identified by BLASTP through multiple alignment of conserved domains. Subsequently, a modeling process of FlhA protein was performed and compared with the protein reported for Oceanibaculum indicum considering the formation of $\alpha$-helixes and $\beta$-sheets. Results showed the presence of some amino acid sequences of the genes related to bacterial flagellar system as MotA FliG, FliN, FliL FlhA, FlhB, FliQ FliR and FliF. Finally, these results suggest that Candidatus Hepatobacter penaei is a rickettsial bacterium having a flagellar system, which possibly confers motility and take part in the infection process in hosts such as marine crustaceans.
\end{abstract}

Key words: NHPB, flagellum, shrimp, intracellular bacteria, marine pathogens

\begin{abstract}
Resumen.- La presencia de sistemas flagelares es común en muchas bacterias de vida libre, sin embargo, estos han sido considerados ausentes en bacterias del orden Rickettsiales. Candidatus Hepatobacter penaei es una bacteria Rickettsial de origen marino descrita como un microorganismo con motilidad, propulsada por un sistema flagelar, el cual también facilita la infección. Siendo esta bacteria uno de los principales riesgos de infección en granjas camaronícolas. El objetivo de este trabajo fue determinar la presencia de genes que codifiquen proteínas del sistema flagelar de la bacteria Candidatus Hepatobacter penaei. Para el análisis se utilizó la secuencia genómica la base de datos del GenBank con número de acceso NZ_JQAJ00000000.1, una vez detectados los genes, se dedujeron las secuencias de aminoácidos. Las secuencias de aminoácidos del sistema flagelar se identificaron mediante BlastP, por medio de un alineamiento múltiple de dominios conservados. Posteriormente, se realizó un modelado de la proteína FlhA y se comparó con la proteína reportada para Oceanibaculum indicum para la conformación hojas $\beta$ y $\alpha$ hélices. Los resultados mostraron la presencia de algunas secuencias de aminoácidos relacionadas con los genes del sistema flagelar de la bacteria como MotA, FliG, FliN, FliL FlhA, FlhB, FliQ, FliR y FliF. Finalmente, estos resultados sugieren que Candidatus Hepatobacter penaei es una bacteria tipo Rickettsia que posee un sistema flagelar, el cual posiblemente le confiere motilidad y forma parte del proceso de infección en hospederos como crustáceos marinos.
\end{abstract}

Palabras clave: NHPB, flagelo, camarón, bacteria intracelular, patógenos marinos

\section{INTRODUCCIÓN}

El flagelo bacteriano es una estructura filamentosa, compuesta por más de 20 proteínas y es el sistema motor de la célula bacteriana (Macnab 2003). El filamento es un tubo hueco helicoidal de aproximadamente $20 \mathrm{~nm}$ de espesor; éste tiene un 'codo' o curva pronunciada justo a la salida de la membrana externa; este codo permite convertir el movimiento giratorio del eje en helicoidal. Un eje se extiende entre el codo y el cuerpo basal, pasando por varios anillos de proteínas en la membrana de la célula que actúan de anclaje. Las bacterias presentan 4 de estos anillos: El par externo (anillo L y anillo P), se encuentra a la altura de la pared y membrana celular. El par interno (anillo Sy M conforman el rotor del flagelo), se inserta en la membrana 
citoplasmática al igual que hacia el lado citoplasmático de la membrana. Por debajo del anillo M existe un quinto anillo, llamado anillo C (llave reguladora), que confiere la conmutación del sentido del giro del motor del flagelo al estar en contacto con el canal de protones yel anillo MS que da la movilidad al flagelo (Diószeghi et al. 2004).

Este sistema flagelar es usualmente conservado entre especies y está conformado por un motor giratorio, que es accionado por una diferencia de potencial electroquímico de iones específicos a través de la membrana citoplasmática (Minamino \& Imada 2015). El motor de Salmonella, impulsado por H+ gira a $300 \mathrm{~Hz}$, el motor de Vibrio spp., impulsado por $\mathrm{Na}+$, puede girar hasta los $1700 \mathrm{~Hz}$. El anillo C y el aparato de exportación, muestran propiedades dinámicas para ejercer sus actividades funcionales. En diferentes especies bacterianas se observan varias estructuras adicionales que rodean la estructura conservada del motor. Éste es utilizado para la motilidad de las bacterias y en algunos casos, se relaciona con el sistema de secreción tipo III (Minamino 2014).

La presencia de sistemas flagelares es común en muchas bacterias de vida libre y representan un antiguo rasgo de las Alphaproteobacterias (Boussau et al. 2004). Durante décadas, los flagelos se han considerado ausentes entre las bacterias Rickettsiales, pero esto es cuestionado recientemente cuando los genes flagelares se identificaron en los genomas de Midichloria sp., Odyssella sp., y algunos endosimbiontes tipo Rickettsia (Georgiades et al. 2011, Sassera et al. 2011, Mariconti et al. 2012, Driscoll et al. 2013). Por ejemplo, Jidaibacter sp., contiene 35 genes que codifican las estructuras flagelares, el conjunto más completo encontrado hasta ahora en los genomas de alphaproteobacterias intracelulares obligadas (Schulz et al. 2015).

Entre las bacterias tipo rickettsias en las cuales se han detectado sistemas flagelares, el microorganismo Candidatus Hepatobacter penaei es conocido como un patógeno causante de la necrosis hepatopancreática (NHPB) en crustáceos marinos (Figueroa-Pizano et al. 2014; Martínez-Córdova et al. 2016). Esta se ha identificado en el orden de las rickettsiales debido a que presenta características endosimbióticas al desarrollarse y multiplicarse dentro de las células epiteliales del hepatopáncreas en varias especies de camarones peneidos: Farfantepenaeus aztecus, F. californiensis, Litopenaeus vannamei, L. setiferus y L. stylirostris (Krol et al. 1991, Frelier et al. 1992, Lightner et al. 1992, Lightner 1996). Es así, que estudios morfológicos han revelado su presencia en el citoplasma y en el epitelio tubular del hepatopáncreas de camarones peneidos (Frelier et al. 1992, Lightner et al. 1992).
La bacteria Candidatus Hepatobacter penaei presenta forma de cocobacilo, su tamaño oscila alrededor de $0,32 \mu \mathrm{m}$ de diámetro mostrando una membrana trilaminar, sin flagelos; tiene una sección transversal constricta en la parte central, indicando replicación por fisión binaria (Krol et al. 1991, Frelier et al. 1992). Sin embargo, estudios posteriores a estos describen una bacteria helicoidal con dimensiones de 0,59 $1,18 \mu \mathrm{m}$ de longitud y $0,36 \mu \mathrm{m}$ de ancho (Loy et al. 1996), posee una membrana trilaminar con 8 flagelos en el ápice basal 1 o 2 en la cresta de la hélice (Lightner et al. 1992), considerándose dos diferentes etapas de vida. Krol et al. (1991). También describen una tercera forma de vida, observada ocasionalmente en el hepatopáncreas infectado; esta forma presenta la morfología de las dos variantes, lo que sugiere el desarrollo de la forma replicativa de cocobacilo a la forma elíptica (Lightner et al. 1992).

Asimismo, Candidatus Hepatobacter penaei prevalece como uno de los principales riesgos de infección en granjas camaronícolas. Por tal motivo, es importante conocer e identificar los factores de virulencia que facilitan la infección, como la presencia de un posible sistema flagelar que otorgue motilidad en los organismos hospederos. Esta información es de importancia porque podría ayudar en el diseño de nuevas estrategias para el control de esta bacteria. Es así, que el objetivo de este trabajo fue determinar la presencia de los genes que codifiquen a las proteínas del sistema flagelar de la bacteria Candidatus Hepatobacter penaei.

\section{Materiales Y MÉTODOS}

Para todos los análisis, se utilizó la secuencia genómica depositada en la base de datos del GenBank con número de acceso NZ_JQAJ00000000.1, en 5 draft o contigs ${ }^{1}$, perteneciente a la bacteria Candidatus Hepatobacter penaei.

\section{Predicción de genes y Secuencia de aminoácidos DEDUCIDA}

Para la detección de genes y proteínas codificantes de la bacteria se utilizó el programa Prokaryotic Dynamic Programming Genefinding Algorithm (Prodigal) (Hyatt et al. 2012), en el servidor público PRODIGAL ${ }^{2}$. Cada fragmento de la secuencia genómica de Candidatus Hepatobacter penaei fue cargada al programa en formato fasta, de acuerdo a las instrucciones del servidor. Se utilizó el código de la tabla de traducción para bacterias y arqueas, activando la opción de salida para los genes coordinados con proteínas traducidas, fue así que los resultados presentaron la predicción de los genes y las posibles secuencias proteicas de dichos genes.

\footnotetext{
${ }^{1}<$ http://www.ncbi.nlm.nih.gov /genome/?term=32949>

${ }^{2}<$ http://prodigal.ornl.gov/server.html)>
} 


\section{Análisis de identidad de las secuencias de AMINOÁCIDOS DEDUCIDAS}

Para la búsqueda de proteínas homólogas, se utilizó BlastP para realizar los estudios de similitud, solamente se consideraron las proteínas reportadas por el BLASTP con los siguientes valores mínimos: identidad del $30 \%$, cobertura del $90 \%$ y el valor E menor de 1e-20, utilizando una matriz de sustitución de los bloques de aminoácidos con un 45\% de identidad (BLOSUM45). Se utilizó la base de datos de UniProtKB para las bacterias en el servidor público de UniProt ${ }^{3}$. De los datos obtenidos en el alineamiento se seleccionaron los resultados con mayor identidad.

\section{AnÁlisis de dominios CONSERVAdos}

Se realizó un análisis de dominios conservados de las secuencias de aminoácidos deducidas a partir de la predicción de genes del sistema flagelar de Candidatus Hepatobacter penaei. El algoritmo utilizado consistió en un alineamiento de secuencias de tipo local específico en posición invertida (RPS BLAST), el cual se comparó una secuencia de la colección de secuencias que están disponibles como varias matrices de puntuación en posición específica (PSSMs). Se tomó un valor E con significancia de 0,01 basado en un ajuste de composición. Se utilizó la base de datos con dominios conservados del Centro Nacional para la Información Biotecnológica (NCBI's CDD) (Marchler-Bauer et al. 2015). Los resultados indicaron que el valor E del dominio conservado presentó una secuencia de aminoácidos deducida.

\section{Alineamiento múltiple de las secuencias de AMINOÁCIDOS}

Las secuencias con similitud adecuada fueron alineadas usando CLUSTAL Omega, mediante la base de datos de UniProtKB para bacterias en el servidor público de UniProt ${ }^{3}$. Este programa usa un algoritmo heurístico progresivo para calcular alineamientos múltiples (Sievers et al. 2011). Clustal Omega utiliza árboles de guía sin semillas del modelo de Hidden Markov y técnicas perfil-perfil para generar alineaciones entre 3 o más secuencias (Soding 2005).

De los resultados obtenidos con BlastP, se realizó el alineamiento múltiple de secuencias de aminoácidos que resultaron con mayor identidad. Estos resultados mostraron las secuencias alineadas, marcaron los aminoácidos conservados y los que presentaban características similares de acuerdo a su cadena lateral.

\footnotetext{
${ }^{3}<$ http://www.uniprot.org>

${ }^{4}<$ http://swissmodel.expasy.org/>
}

Modelado y alineamiento de Secuencias de AMINOÁCIDOS EN 3D

El modelado se realizó con la secuencia de aminoácidos deducida de los posibles genes de Candidatus Hepatobacter penaei correspondiente a la proteína FlhA y la secuencia de la proteína de referencia con mayor identidad obtenida con BlastP. Dichas secuencias se introdujeron en el portal de Swiss Model ${ }^{4}$ y se utilizaron proteínas previamente caracterizadas como molde para realizar el análisis (proteína FlhA de Salmonella sp.), obteniendo los modelos en 3D (Biasini et al. 2014). Estos fueron analizados en el portal Matras (<http:// strcomp.protein.osaka-u.ac.jp/matras $>$ ), para realizar una comparación de la presencia del $\alpha$-hélice y las hojas $\beta$, mediante un algoritmo de alineamiento jerárquico basado en el modelo de transición de la evolución de Markov (Kawabata \& Nishikawa 2000). Los resultados mostraron la similitud entre ambos modelos de las secuencias de aminoácidos de la proteína FlhA en la conformación del $\alpha$-hélices y las hojas $\beta$.

\section{Resultados}

El análisis de los 5 fragmentos del genoma de Candidatus Hepatobacter penaei permitió detectar un total de 1067 posibles genes. Las secuencias de aminoácidos deducidas fueron analizadas mediante BlastP mostrando identidades y homología para las distintas proteínas de alphaproteobacterias, como proteínas implicadas en el metabolismo, replicación, transcripción y traducción de Candidatus Hepatobacter penaei (datos no mostrados). Dentro de estas secuencias se obtuvieron 12 secuencias con alta similitud con las proteínas del sistema flagelar. Las proteínas encontradas corresponden a componentes del motor (MotA, FliGC-terminal), la base (FliF, FliN, FliG N-terminal), la maquinaria de exportación (FlhB, FliQ, FliR, FlhA, FliL), el eje de transmisión (FlgG), el gancho y los adaptadores (FlgF, FlgK) (Fig. 1).

Una secuencia de aminoácidos encontrada en el genoma de Candidatus Hepatobacter penaei codificó 285 aminoácidos (Fig. 2). Este péptido mostró una similitud con las proteínas tipo MotA, con un valor E de 1e-99 aminoácidos, una cobertura cercana al 98\% (Tabla 1); el análisis de los dominios confirmó que esta proteína corresponde a la secuencia proteica MotA (Tabla 2). Esta proteína forma un canal de iones $(\mathrm{H}+, \mathrm{Na}+)$ (Minamino \& Imada 2015) y se encuentra unida a otra proteína MotB, permitiendo el paso de los iones desde la capa de peptidoglicanos hasta la membrana interna (Morimoto \& Minamino 2014). Este canal provee los iones necesarios para la activación del aparato exportador, debido a su arreglo en forma de F0F1ATPasa. Sin embargo, en la secuencia correspondiente para la proteína MotB no se encontró en las secuencias reportadas en el genoma de CHP. 


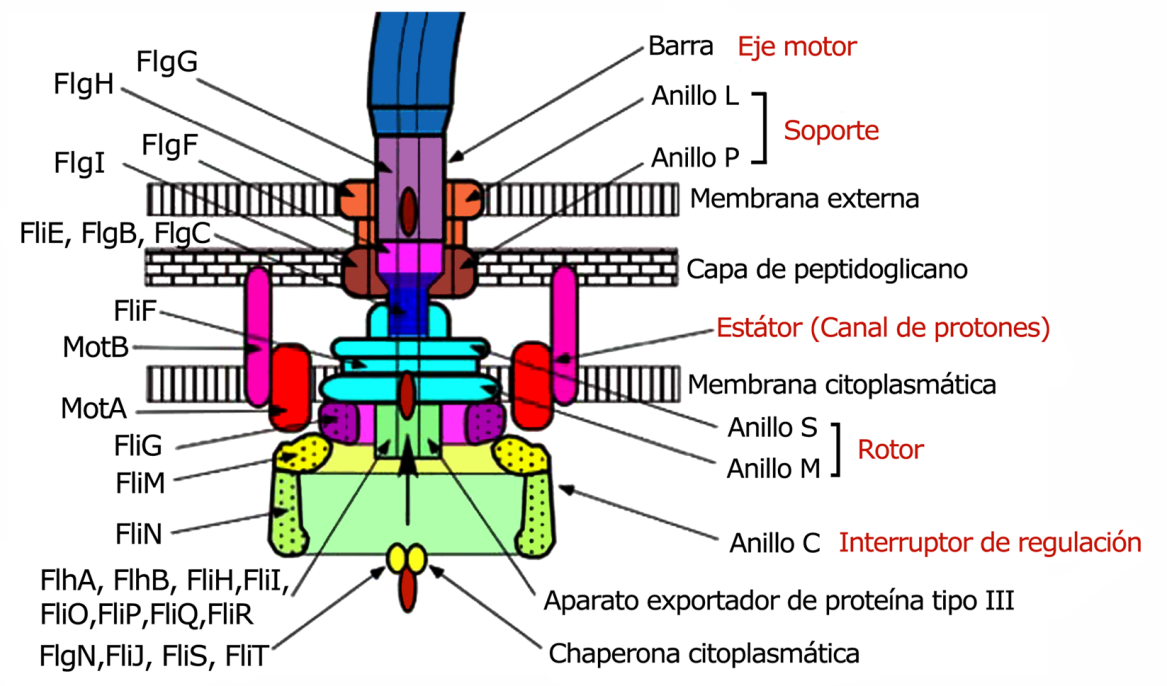

Figura 1. Estructura del sistema flagelar (Pallen \& Matzke 2006) / Flagellar system structure (Pallen \& Matzke 2006)

Desafortunadamente, no se conoce que proporción del genoma falta y cabe la posibilidad que el gen MotB se encuentre entre lo faltante. Aunque la ausencia de MotB no afecta el ensamble del flagelo, es importante precisar la existencia del gen MotB porque en su ausencia afecta la fuerza del protón motriz al inhibirse en el canal de iones resultando un flagelo sin movilidad, afectando esto a la motilidad y adhesión de la bacteria (Morimoto et al. 2013).

Existen 4 aminoácidos altamente conservados en la proteína MotA, los cuales fueron identificados en la secuencia de la proteína deducida. La Val35 de MotA interactúa con el Asp33 de MotB para la formación del canal hidrofóbico por el cual se da el flujo de protones (Braun et al. 2004). De igual manera, la Pro173 de MotA, tiene la función de anclaje con Asp33 de MotB asegurando de esta manera el funcionamiento del motor del sistema flagelar (Nakamura et al. 2009). La presencia de ambos aminoácidos en la secuencia deducida corresponde a MotA apoya la idea que el gen codificante para la proteína MotB se encuentre en el genoma de Candidatus Hepatobacter penaei, aunque en este estudio no se logró identificar.

De igual forma, Arg90 y Glu98 son aminoácidos altamente conservados (Morimoto et al. 2010) y ambos fueron detectadas en la proteína deducida MotA de Candidatus Hepatobacter penaei. La sustitución de estos aminoácidos por mutaciones puede tener un efecto negativo en el motor y con ello en la motilidad de la bacteria, debido a que tiene un efecto directo en la rotación del flagelo. Esto se debe a que Arg90 y Glu98 de MotA, presentan interacciones electroestáticas con los aminoácidos de la proteína FliG en la cadena C-terminal. El
Aspartatoen la posición 289 (Asp289) y Arginina en la posición 281 (Arg281) del FliG, son aminoácidos que interactúan con Arg90 y Glu98 de MotA, respectivamente (Morimoto et al. 2013). La presencia de estos aminoácidos en las secuencias deducidas para MotA y FliG de Candidatus Hepatobacter penaei es de importancia en el sistema flagelar para la motilidad de la bacteria, por ser uno de los factores de virulencia más usados en la invasión al hospedero.

La base del sistema flagelar está conformada por el anillo C (complejo interruptor) ( $\mathrm{FliG}_{26}, \mathrm{y}\left[\mathrm{FliM}_{1} / \mathrm{FliN}_{3}\right]_{37}$ ) y el anillo MS $\left(\right.$ FliF $\left._{26}\right)$ (Morimoto \& Minamino 2014). De los cuales, se lograron identificar en este estudio 3 secuencias de aminoácidos deducidas de la predicción de genes con características similares a las proteínas FliG, FliN y FliF. Se encontró una secuencia de 379 aminoácidos con identidades entre el 34 y $38 \%$ en FliG, con aminoácidos de características similares mayores al 60\%, con una cobertura igual o mayor al $95 \%$ y un valor E mínimo de 1.1e-66 (Tabla 1). Esto sugiere una posible relación con la proteína FliG, lo cual fue reforzado con el análisis de dominios conservados, en que se determinó que se trata de una proteína homóloga de FliG (Tabla 2).

El complejo interruptor actúa directamente con los canales de protones de la transmembrana, especialmente en la generación de la fuerza de torsión, en la conmutación de rotación y en el ensamblaje flagelar (Sircar et al. 2014). Asimismo, el complejo FliG se encuentra más cercano a la membrana y participa directamente en la generación de la fuerza de torsión, interactuando en el C-terminal con MotA (descrito anteriormente) y en el N-terminal con FliM. El FliM se encuentra 


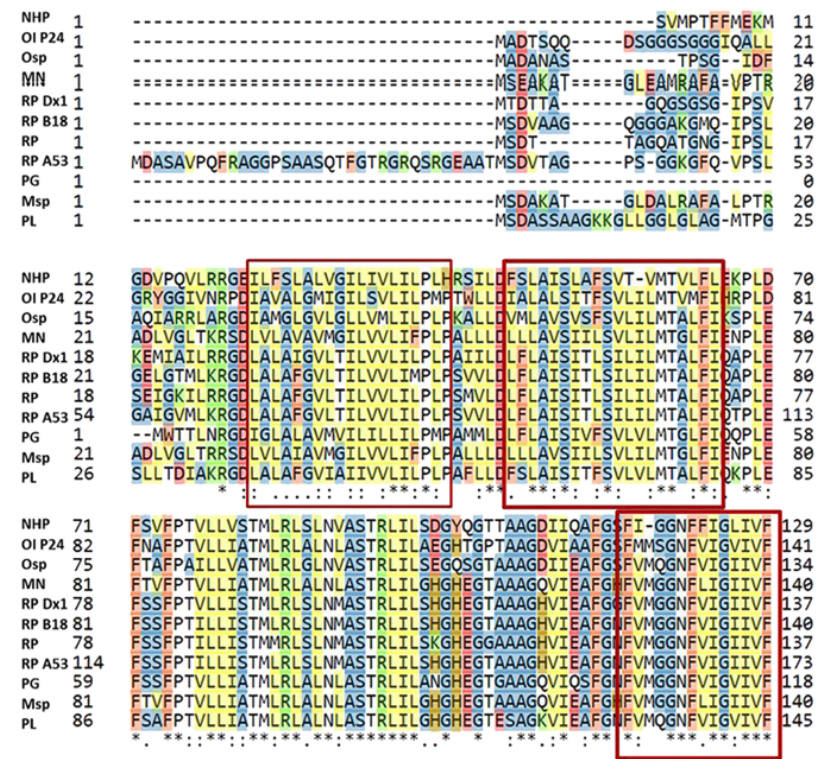

NHP 130 AILWINFW TKGSGRIAEWARFSLDALPGKQMSVDADLSSGAIDQ-EAKVRRAHIQE 188

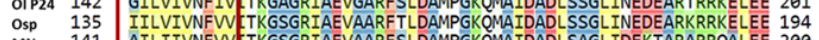

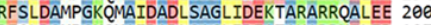
$\begin{array}{lll}\text { RP B18 } & 141 & \text { AILVIVNFW TKGSGRIAEVAARFQLDSMGKQMAIDADLSAGLIDEATAKARRKELED } 197 \\ & \end{array}$

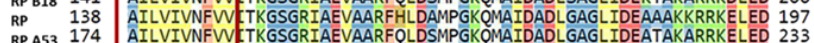
RPA53 174 PG 119 Msp 146

$\begin{array}{ll}\text { NHP } & 189 \\ \text { OI P24 } & 282\end{array}$ $\begin{array}{ll}\text { Osp } & 195 \\ \text { MN } & 201\end{array}$ RP DX1 19 \begin{tabular}{ll} 
RP B18 & 201 \\
RP & 198 \\
\hline$P G 53$ & 234
\end{tabular} $\begin{array}{lll}\text { RPAS3 } & 234 \\ \text { PG } & 179\end{array}$ Msp 201

$\begin{array}{ll}\text { NHP } & 248 \\ \text { OI P24 } & 262\end{array}$ $\begin{array}{ll}\text { Osp } & 255 \\ \text { MN } & 261\end{array}$ RP Dx1 258 \begin{tabular}{ll} 
RP B18 & 261 \\
RP & 258 \\
\hline RPA53 & 294
\end{tabular} RPA53 294 $\begin{array}{ll}\text { Msp } & 261 \\ \text { PL } & 266\end{array}$ OI P24 322 LPFALLAAITGGAAYLLTRQKKAETLRKAE----.-AVATETAEMPVAEEPISTALHIDLIR 434 $\begin{array}{llll}\text { Osp } & 315 & \text { IPFGLMAAGAGALAWTSAQRREKAVETEAREALAEEEAAAQGPDPTAPIEDTLHIDELK } & 431 \\ \text { MN } & 321 & \text { LPFLVLAGGAAFLARRIARLPKAAD-.-PEAAAPGA-.--AAAPEETVSDLLRLDDLK } 431\end{array}$ $\begin{array}{lll}M N & 321 & \text { LPFLVLAGGAAFLARRIARLPKAAD-.--PEAAAPGA----AAAPEETVSDLLRLDDLK } 431 \\ \text { RP DX1 } & 318 \text { LPFLALGGGAGYLAFKAGKRKAQVSAEAAQAVAPEMAAAAAAAAEEPIAAALRIDDLK } 435\end{array}$ RP B18 321 LPFLLLGGGAAWLAVSAGKRNRAAKVTEEREANAPAAAAAAASAAEEEPIQASLKIDDLK 438

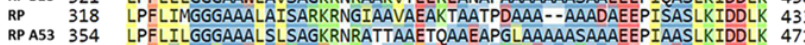
PG 299 LPFLALAGLSGWLAFKSSDTKKQTAMKIAE----AKAIETAPKPAPEPPITDALKMDELR 414 $\begin{array}{llll}\text { Msp } & 321 & \text { LPFLLLAGGAAYLARRIARMPKPVD-.-PEAPAAGGAPGAGAQPKEETVADLLRLDDLK } & 435 \\ \text { PL } & 326 & \text { VPFLALAGATGGLAFYTKSRRERADEIAV--.-AEAKEADAAPVAEEPIGTALKMDELR } & 438\end{array}$

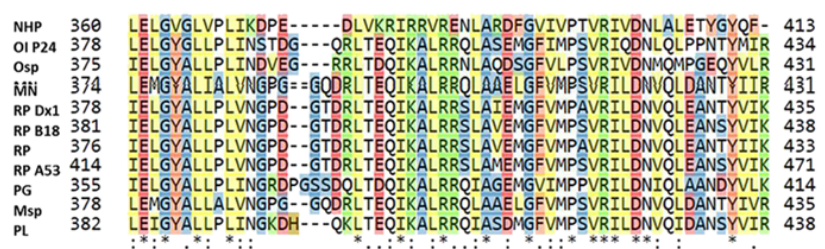

NHP 414 VKEVKAAAGTIFPDKVIALDPDGSLEKIPGEAGKEPVLGIPGIWIHASELKQAKEKGYV- 472 $\begin{array}{llll}\text { OI P24 } & 435 & \text { VKEIESGRGDIRPNMLLVMDPRGQKIDLPGENTREPTFGLPAMINPSHREEMMRGYTV } & 494 \\ \text { Osp } & 432 & \text { VKEMEAGVGELKINOTLAMNPSGAOVELPGTHVEPAFGLATWIDESNREATTRGYTL } 491\end{array}$ MN 432 VKEIEAGTGQIFPGQYMAMDPMGGQVQLPGQHLLEPTFGLPATWIDASLRDQAQLRGYTV 491 RP DX1 436 IKEVDAGSGRIWPNQFMVMDPAGEQVTVPGIHTTEPTFGLPATWVDANFKEEASLKGYTV 495 $\begin{array}{lll}R P & 439 & \text { IKEVDAGTGRIWPSQFMVMDPGGNQVAVPGIHTT EPTFGLPATWVDASLKEEATMKGYTV } 498 \\ R P & 434 & \text { IKEVDAGOGRIWPSOFMVMDPGGNOVSVPGIHTIEPTFGLPATWVDASLKEEATMKGYTV } 493\end{array}$ $\begin{array}{lll}\text { RP } & 434 & \text { IKEVDAGQGRIWPSQFMVMDPGGNQVSVPGIHTIEPTFGLPATWVDASLKEEATMKGYTV } \\ \text { RP A53 } & 472 & \text { IKEVDAGTRRIWPNQFMVMDPGGAQVTVPGIHTI }\end{array}$ PG 415 VKEV $\begin{array}{llll}\text { Msp } & 436 & \text { VKEIEAGTGQVFPGQFMAMDPMGGQVLPGQHLLEPTFGLPATWIDASLRDQAQRGYTV } 495 \\ \text { PL } & 439 & \text { VKEVESGRGDVVGQLLVDPHGSQIQLPGLHTTEPAFGLPATWIDEGLREASFRGYTV } 498\end{array}$ NHP 473 ADAKALLMTHITETIKKHLADLFSFSLQQSIDSLRKPYQKMYGDMVPMQISAASVGRI- 531

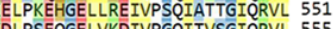
$\begin{array}{lll}\text { RP DX1 } & 496 & \text { VDAATVLSTHLTELLKANMSDLLSYGETQKLLKDLPSEQGELVKDIVPGQITVSGIQRVL } 555 \\ \text { RP B18 } & 499 & \text { VDAATV STTL }\end{array}$ RP 494 RP A53 532 $\begin{array}{ll}\text { PG } & 475 \\ \text { Msp } & 496 \\ & 499\end{array}$ $\begin{array}{ll}\text { Msp } & 496 \\ \mathrm{PL} & 499\end{array}$ SATVLSTHLTELLLKANVADLLSYGETQKLLKDLPKEQGELVKDIVPAQITVGIQRVL 553
591 VDPATVLSTHLTETIKTNIAELLTYADVQKLLKELKGEOAKLVDDLIPSOITVSGVQRVL 534
VDAATWSTHLTEVIKAHVSELLNHVEVOKLLRELPKEHTELLKETVPSTATTGIORVL 555 VDPPTVLTTHLTEIIKDNMADLLSYAEVQKLLDELGKEHKKLVEDLVPSQITVTGIQRVL 558

NHP 532 KNLLKEQVSIRDLGTIIEAISESLSYTKEPDAIGERVRMSLARQIASPFLKDGQLWAL -- 589 OI P24 555 QNLLSERISIRDLPTILEGVSEASTYTRNITMITEHVRSRLARQISEQYVNEEGHIPIVT 614 OsP 552 QNLLKERSIRDLAAIIEGIAEASOATNNLDAMTEHVRRLARQICYANRGPONLPVLS 611 RP DX1 556 QYLLAERVSIRDLGTIVEGIAEVAGHVKNPRDIVEHVRARLGRQICAQYQGPGGVPIIT 611 RP B18 559 O RP 554 QLLLAERISIRDLSTILEGVADALAISRNPSTIVEHVRARLARQICAONTSYNGYLPLIA 613

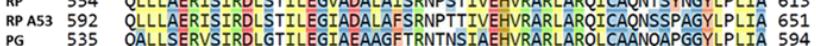
$\begin{array}{lll}\text { PG } & 535 & \text { QALLSERVSIRDLGTILEGIAEAAGFTNTNSIAEHVRARLARQLCAANQAPGGYPLIA } 594 \\ \text { Msp } & 556 & \text { OYLLAERVSIRDLGTIVEGIAEVAGHVKNPRDIVEHVRARLGROICAOYOGPGGLPIIT } 615\end{array}$

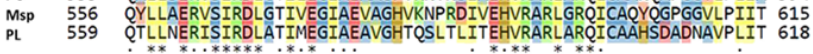
$\begin{array}{lll}\text { NHP } & 590 \\ \text { OIP24 } & 615 \\ 0.5 & 612 & 15\end{array}$

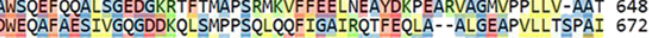

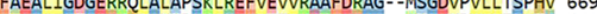
RP DX1 616 LSAKWEQAFAESIIGQGEDRTLAMAPSKLSEFMTAVRDKFEQAA--REGEAPVLVTSATI 673 RP B18 619 LSAKWEPAFAESTGOGDRSLAMPSKLSEMMTTVRKFEOAA-REGEAPVLVTSST 671 $\begin{array}{lll}R P & 614 \\ R P A 53 & 652 & \text { LSAKWEQAFAESIIGOGEERSLAMOPSKLSEFMTTVRKFEQAA--REGEAPVLVTSASI } 709\end{array}$ PG 595 LSPKWEQAFLESIIGEGDDRQLAMQPSKLQEFVGLVRDSFEKAA--QQGEVPVLLTSPQS 652 $\begin{array}{lll}\text { Msp } & 616 & \text { LSPAWESAFLDAIVGQGDERHLAMQPSRLSEFVTTVRRFEEAA--RMGEMPVLVTSVQA } 673 \\ \text { PL } & 619 & \text { LSPRWEQAFAESLVGAGEEKQLAMQPSVLQDFIANVRDRFEDAA--RAGEVPVLLTSPGV } 676\end{array}$ NHP 649 LPYVRSLSEKTRPDIAVLSDAEATSHYRVHIVGEV 683 OIP24 673 RPYVRSIIERFRPATVLSQSEIHPKAKIKTLGQI 707 Osp 670 RPY MN 670 RPFVRSIIERFRRETPVMSAEIHPRARLKTVGSV 704 RPFRSIVERFR RP 672 RPFVRSLVERFRAQTTVLSQAEIHPRARLKTVGSV 706 RPAS3 710 RPFVRSLVERFRAQTTVLSQAEIHPRARLKTVGSI 744

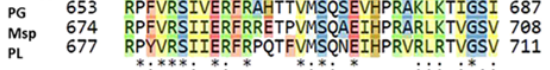


Tabla 1. Similitud de proteínas homólogas del sistema flagelar de Candidatus Hepatobacter penaei / Similarity of homologous proteins of the Candidatus Hepatobacter penaei flagellar system

\begin{tabular}{|c|c|c|c|c|c|}
\hline Proteína & Especies & Valor E & $\begin{array}{l}\text { Identidad } \\
(\%)\end{array}$ & $\begin{array}{l}\text { Similitud } \\
(\%)\end{array}$ & $\begin{array}{c}\text { Cobertura } \\
(\%)\end{array}$ \\
\hline \multirow{5}{*}{ MotA } & Desvosia riboflavin & $7.6 \mathrm{e}-102$ & 54,1 & 72,8 & 98 \\
\hline & Rhodospirillum rubrum & $2.1 \mathrm{e}-101$ & 51,2 & 72,8 & 99 \\
\hline & Skermanella stibiiresistens & 19.e-99 & 51,2 & 72,8 & 99 \\
\hline & Pelagibacterium halotolerans & $2.8 \mathrm{e}-99$ & 51,6 & 72,4 & 98 \\
\hline & Candidatus paracaedibacter acanthamoebae & $7.7 \mathrm{e}-99$ & 51,1 & 71,8 & 98 \\
\hline \multirow{5}{*}{ FliG } & Micavibrio aeruginosavorus $\mathrm{EPB}$ & $2-e 72$ & 38,3 & 62,9 & 95 \\
\hline & Phenylobacterium zucineum & $2.7 \mathrm{e}-70$ & 36,8 & 66,0 & 96 \\
\hline & Thalassopira profundimaris & $2.7 e-69$ & 35,9 & 64,1 & 96 \\
\hline & Azospirillum sp CAG:239 & $4.4 \mathrm{e}-68$ & 35,5 & 62,0 & 97 \\
\hline & Oceanibaculum indicum $\mathrm{P} 24$ & $1.2 \mathrm{e}-67$ & 34,0 & 63,5 & 96 \\
\hline \multirow{5}{*}{ FliF } & Oceanibaculum indicum & $3.3 \mathrm{e}-121$ & 39,8 & 59,9 & 98 \\
\hline & Magnetospira sp. & $3.4 \mathrm{e}-119$ & 40,2 & 62,8 & 98 \\
\hline & Azospirillum sp. & $1.2 \mathrm{e}-115$ & 38,7 & 60,4 & 99 \\
\hline & Rodopseudomonas palustris DX1 & $3.1 \mathrm{e}-115$ & 40,2 & 59,8 & 99 \\
\hline & Desvosia sp. & $3.7 \mathrm{e}-111$ & 40,8 & 58,8 & 99 \\
\hline \multirow{5}{*}{ FliN } & Caenispirillum salinarum AK4 & $5.5 \mathrm{e}-21$ & 40,9 & 66,6 & 99 \\
\hline & Candidatus phaemarinobacter ectocarpi & $2.6 \mathrm{e}-20$ & 40,9 & 59,8 & 99 \\
\hline & Rhodospirillum centenum & $5.0 \mathrm{e}-20$ & 47,6 & 64,1 & 76 \\
\hline & Candidatus Paracaedibacter acanthamoeba & $5.7 e-20$ & 41,1 & 62,9 & 99 \\
\hline & Acetobacter sp. & $6.2 \mathrm{e}-20$ & 35,5 & 64,5 & 94 \\
\hline \multirow{5}{*}{ FlhB } & Caenispirilum salinarium & $2.5 \mathrm{e}-64$ & 38,4 & 57,7 & 96 \\
\hline & Phaeospirillum molischianum & $6.4 \mathrm{e}-64$ & 37,4 & 59,2 & 99 \\
\hline & Candidatus arcanobacter lacustris & $6.7 e-60$ & 34,4 & 58,1 & 99 \\
\hline & Magnetospirillum magnetotacticum & $1.2 \mathrm{e}-59$ & 35,1 & 58,4 & 98 \\
\hline & Azospirillum sp. CAG:239 & $2.8 \mathrm{e}-58$ & 35,7 & 58,1 & 97 \\
\hline \multirow{5}{*}{ FlhA } & Oceanibaculum indicum P24 & 0 & 48,0 & 69,4 & 98 \\
\hline & Oceanicaulis sp. & 0 & 50,0 & 70,1 & 97 \\
\hline & Methylobacterium nodulans & 0 & 47,6 & 67,7 & 100 \\
\hline & Rhodopseudomonas palustris DX-1 & 0 & 48,1 & 68,5 & 97 \\
\hline & Polymorpum gilvum & 0 & 48,7 & 69,0 & 97 \\
\hline \multirow{5}{*}{ FliQ } & Maricaulis maris & $1.5 \mathrm{e}-26$ & 55,3 & 77,6 & 93 \\
\hline & Brevundinomas abyssalis & $8.6 \mathrm{e}-26$ & 55,3 & 76,5 & 93 \\
\hline & Asticcacaulis benevestitus & $9.6 \mathrm{e}-25$ & 49,4 & 74,7 & 95 \\
\hline & Hirschia baltica & $1.5 \mathrm{e}-23$ & 50,6 & 72,9 & 92 \\
\hline & Afipia sp. & $2.1 \mathrm{e}-23$ & 52,9 & 70,6 & 92 \\
\hline \multirow{5}{*}{ FliR } & Magnetospirillum sp. SO-1 & $1.5 \mathrm{e}-30$ & 32,3 & 52,0 & 99 \\
\hline & Candidatus paracaedibacter acanthamoebae & $1.5 \mathrm{e}-28$ & 30,6 & 51,4 & 98 \\
\hline & Phaeospirillum fulgum & $3.0 \mathrm{e}-28$ & 30,0 & 51,6 & 94 \\
\hline & Afipia sp. & $2.3 e-27$ & 33,5 & 52,6 & 94 \\
\hline & Thalassopira profundimaris & $1.6 \mathrm{e}-25$ & 30,3 & 48,2 & 100 \\
\hline \multirow{5}{*}{ FliL } & Oceanibaculum indicum & $1.1 \mathrm{e}-22$ & 41,2 & 67,5 & 75 \\
\hline & Phaeospirillum molischianum & $5.4 \mathrm{e}-21$ & 41,3 & 62,8 & 77 \\
\hline & Magnetospirillum magneticum & $2.8 \mathrm{e}-20$ & 42,2 & 62,1 & 76 \\
\hline & Magnetospirillum magnetotacticum & $2.8 \mathrm{e}-20$ & 43,1 & 62,9 & 76 \\
\hline & Phaeospirillum fulgum & $1.1 \mathrm{e}-19$ & 40,2 & 59,0 & 77 \\
\hline
\end{tabular}


Tabla 2. Presencia de dominios conservados de las proteínas del sistema flagelar de Candidatus Hepatobacter penaei / Presence of conserved domains of the proteins the Candidatus Hepatobacter penaei flagellar system

\begin{tabular}{lcc}
\hline Proteína & Intervalo & Valor E \\
\hline MotA & $1-283$ & $7.44 \mathrm{e}-138$ \\
FliG & $14-339$ & $4.54 \mathrm{e}-81$ \\
FliF & $3-532$ & $1.56 \mathrm{e}-171$ \\
FliN & $1-124$ & $7.82 \mathrm{e}-28$ \\
FlhB & $1-362$ & $1.41 \mathrm{e}-101$ \\
FlhA & $4-695$ & $0 \mathrm{e}+0$ \\
FliQ & $1-85$ & $5.23 \mathrm{e}-27$ \\
FliR & $1-208$ & $1.97 \mathrm{e}-25$ \\
FliL & $36-152$ & $7.79 \mathrm{e}-22$ \\
\hline
\end{tabular}

en el centro del rotor e interactúa con el CheY, que es una proteína de señalización fosforilada (CheY-P). Por otro lado, FliN reside en el extremo citoplásmico del rotor y es esencial para la exportación flagelar, montaje y posiblemente para la conmutación.

De la misma manera se determinó la presencia de una proteína FliF en el genoma de Candidatus Hepatobacter penaei, presentó semejanzas entre $37-41 \%$, con un porcentaje de aminoácidos positivos hasta en un $60 \%$, con una cobertura de $95-99 \%$ y junto al dominio FliF con intervalos de aminoácidos de 3-532 (Tabla 1).

El sistema regulador del flagelo corresponde al anillo $\mathrm{C}$ (FliN, FliG, FliM) y al rotor MS (FliF). De estas 4 proteínas se lograron identificar 3 de ellas (FliN, FliG y FliF). El anillo MS está formado por 26 copias de FliF y actúa como una plataforma de montaje (Marimoto \& Minamino 2014). Se utilizó el anillo de MS como una plantilla, FliG, FliM y FliN y se ensambló para formar el anillo $\mathrm{C}$ en la cara citoplásmica del anillo MS. FliG se asoció directamente con la cara citoplásmica del anillo de MS con una estequiometría FliG 1: 1 FliF, quedando el anillo FliG con una simetría de 26 veces. Por su parte, el sistema FliG contiene 3 dominios: FliG N, FliG FliGN, los cuales se unen directamente al anillo MS (Levenson et al. 2012). FliG está directamente implicada en la interacción con una proteína estátor MotA; lo que sugiere que FliG proporciona un sitio de unión para FliM (Vartanian et al. 2012). FliM y FliN forman un complejo estequiométrico estable FliM 1: 4 FliN y ocupan la mayor parte del anillo C. Ciertas mutaciones en FliG, FliM y FliN afectan a la conmutación y rotación, lo que indica que estas 3 proteínas son responsables de la dirección de rotación del motor (Marimoto \& Minamino 2014).
La proteína FlhA forma parte del aparato exportador del flagelo (Fig. 1). Esta proteína se logró identificar en la bacteria Candidatus Hepatobacter penaei mediante un BlastP del cual se pueden observar los resultados en la Tabla 1, mostrando identidades entre el 47-50\% y aminoácidos positivos de 68$70 \%$. En particular, NHPB mostró una identidad del 48 y un 69,4\% de similitud en su secuencia de aminoácidos de Oceanibaculum indicum. Esto indica la posibilidad de que esta proteína codificante de Candidatus Hepatobacter penaei corresponda a una proteína homóloga del sistema flagelar FlhA. Además, en la Tabla 2 se muestra la presencia del dominio de la proteína FlhA, lo cual confirma que se trata de esta proteína flagelar. Se realizó un modelado de la estructura proteica de FlhA de Candidatus Hepatobacter penaei y Oceanibaculum indicum en 3D mediante el programa Swiss Model utilizando como molde una proteína FlhA de Salmonella. Se obtuvieron los modelos en 3D y se realizó un alineamiento de la estructura entre ambos modelos utilizando Matras 1.2, observándose una conformación similar en las hojas $\beta$ y $\alpha$-hélices correspondiendo ambas una estructura similar (Fig. 3).

Durante el análisis de las proteínas codificantes de Candidatus Hepatobacter penaei, se observó similitud con la proteína FlhB correspondiente al aparato exportador del sistema flagelar (Tabla 1). Esta proteína con 362 aminoácidos posee regiones inter-membranales que sirven de anclaje del sistema flagelar y dominios citosólicos. Los resultados mostraron una identidad de entre 30-38\%, con aminoácidos positivos entre $54-60 \%$, una cobertura entre el $97-99 \%$ y un valor de E de 1e-64. Esto evidencia la relación existente entre dicha proteína con proteínas homologas de FlhB, por lo cual se realizó un análisis de dominios. Esta proteína presentó dominios correspondientes a la proteína FlhB, lo cual indicó que se trataba de una proteína homóloga.

Una proteína de 152 aminoácidos presentó similitud con proteínas FliL, la cual correspondió al sistema exportador como parte del sistema rotor. Los resultados del BlastP mostraron una identidad entre 40-43\%, con un porcentaje de aminoácidos entre 59-67, una cobertura entre 71-77\% y valor E aproximado a los 2.8e-20 (Tabla 1). Si bien el porcentaje de cobertura es relativamente bajo, esta región se encuentra dentro del dominio de FliL, el cual se encuentra a partir del aminoácidos 36 hasta el 152; indicando que se trata de proteínas homólogas correspondientes a la proteína FliL. Asimismo, esta proteína formó un anillo con 6 proteínas unidas a la proteína FliJ, conformando la parte citosólica del sistema exportador flagelar.

Otra proteína conformada por una secuencia de 92 aminoácidos presentó similitud con FliQ, con una identidad entre 49-55\%, un porcentaje de aminoácidos positivos entre 70$77 \%$, una cobertura alrededor del $90 \%$ y un valor aproximado 
de E igual a 1.5e-23 (Tabla 1). El análisis de dominios mostró la presencia del dominio FliQ en el intervalo de aminoácidos del 1-85, lo cual evidenció la presencia de esta proteína en el genoma de Candidatus Hepatobacter penaei.

También, se encontró una secuencia de aminoácidos deducida con la predicción de los genes de Candidatus Hepatobacter penaei compuesta de 218 aminoácidos con una similitud-de proteínas FliR. Para esta identidad se registró al alrededor del $30 \%$, con aminoácidos positivos en un $50 \%$ y una media en la cobertura del 94\%. Las proteínas FliQ y FliR, son proteínas intermembranales del aparato exportador junto con las proteínas FliO y FliP, las cuales no fueron identificadas en las proteínas codificantes en este estudio. Sin embargo, la presencia de las proteínas FliQ y FliR representó una posible evidencia de la presencia de este sistema en el genoma de la bacteria (Minamino 2014).

\section{Discusión}

Las proteínas asociadas al sistema flagelar han sido estudiadas en diversos tipos de bacteria. Por ejemplo, Foynes et al. (1999) documentaron un efecto negativo sobre la adherencia Helicobacter pylori en ratones después de haber silenciado el gen que codifica para FliQ. Se cree que esto pudo deberse a que esta proteína afectó al aparato exportador, evitando que los factores de adherencia fueran exportados al exterior de la célula, impidiendo con ello la adherencia a las células epiteliales. Por otra parte, en otro estudio se analizó el efecto de FliL sobre la motilidad de la bacteria en nado y en enjambre; observándose que el nado de la bacteria no fue afectado por el silenciamiento de FliL; sin embargo, la motilidad tipo enjambre si fue afectada (Attmannspacher et al. 2008). Lo anterior se pudo deber a que el cambio de protones no se afecta, otorgando movimiento al flagelo en el modo de nado; mientras que en el a

C

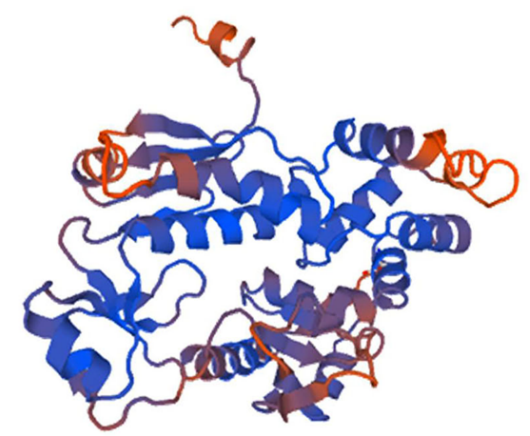

b

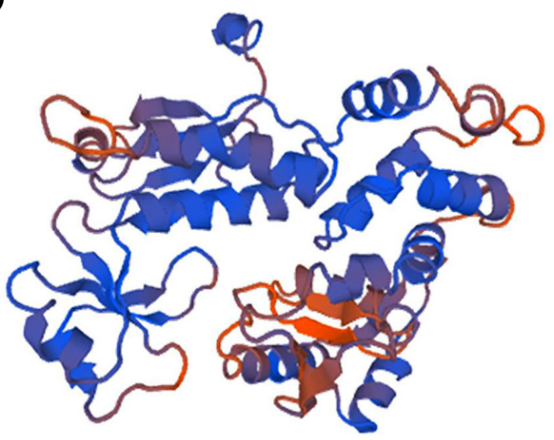

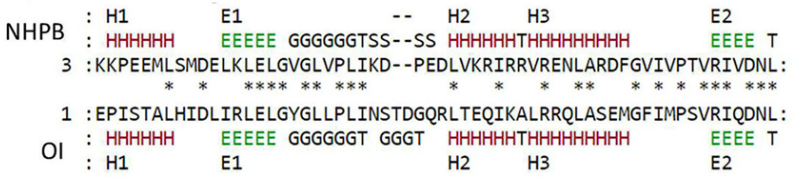

Figura 3. Modelado de los dominios citosólicos de proteínas homologas de FlhA. a) FlhA de NHPB, b) FlhA de Oceanibaculum indicum. c) Alineamiento de los modelos de Candidatus Hepatobacter penaei y $\mathbf{O}$. indicum. $\mathbf{H}, \alpha$ hélice; E, hojas $\beta$ / Modeling of cytosolic domains FlhA proteins homologous. a) FlhA of NHPB, b) FlhA of Oceanibaculum indicum, c) Alignment Candidatus Hepatobacter penaei and O. indicum models. H, $\alpha$-helix; E, $\beta$-sheet 
modo de enjambre, se cree que esta proteína puede estar relacionada con los filamentos que sirven de enganche, debido a que FliL se presenta en la parte citosólica de la bacteria otorgándole resistencia a dichos filamentos (Suvanachuti 2015).

FlhA y FlhB corresponden a proteínas con regiones intermembranales y citosólicas, las cuales regulan el paso de las sustancias exportadas a través del sistema flagelar (Minamino \& Macnab 2000). Éste se regula debido a una región muy conservada (NPTH) presente entre 2 dominios de FlhB el cual en presencia del sustrato específico aumenta las fuerzas electrostáticas y sufre escisión entre ambos dominios de FlhB, lo cual resulta en la activación del sistema exportador (Ferris et al. 2005). Si bien durante el estudio no se encontraron las proteínas codificantes para el aparato exportador completo, los resultados obtenidos revelan de la presencia de este sistema, el cual resulta importante para el sistema de secreción tipo III. Este aparato exportador presenta similitudes con una $\mathrm{F}_{0} \mathrm{~F}_{1}$ ATPasa (Minamino 2014).

En conclusión, las secuencias de aminoácidos deducida de Candidatus Hepatobacter penaei, descritas anteriormente relacionadas con el sistema flagelar proporcionan información acerca de uno de los posibles factores de virulencia utilizados por esta bacteria. La presencia de flagelos en algunas etapas de su ciclo de vida de esta bacteria indica que la motilidad puede ser necesaria aun para las bacterias intracelulares obligadas como las del tipo rickettsia. Si bien, este estudio evidencia un posible factor de virulencia relacionado con la motilidad de la bacteria, adhesión e invasión en el hospedero, es necesario evidenciar todos los factores de virulencia presentes, con la finalidad de establecer alternativas para el control de esta bacteria.

\section{Agradecimientos}

Especial agradecimiento al Consejo Nacional de Ciencia y Tecnología por el apoyo recibido a través del Proyecto 168614.

\section{LITERATURA CITADA}

Attmannspacher U, BE Scharf \& RM Harshey. 2008. FliL is essential for swarming: motor rotation in absence of FliL fractures the flagellar rod in swarmer cells of Salmonella enterica. Molecular Microbiology 68: 328-341.

Biasini M, S Bienert, A Waterhouse, K Arnold, G Studer, T Schmidt, F Kiefer, TG Cassarino, M Bertoni, L Bordoli \& T Schwede. 2014. SWISS-MODEL: modelling protein tertiary and quaternary structure using evolutionary information. Nucleic Acids Research 42: 252-258.

Boussau B, EO Karlberg, AC Frank, BA Legault \& SG Andersson. 2004. Computational inference of scenarios for $\alpha$-proteobacterial genome evolution. Proceedings of the National Academy of Sciences 101:9722-9727.
Braun TF, LQ Al-Mawasawi, S Kojima \& DF Blair. 2004. Arrangement of core membrane segments in the MotA/MotB protein-channel complex of Escherichia coli. Biochemistry 43:35-45.

Diószeghy Z, P Závodszky, K Namba \& F Vonderviszt. 2004. Stabilization of flagellar filaments by HAP 2 capping. FEBS Letters 568: 105-109.

Driscoll T, JJ Gillespie, EK Nordberg, AF Azad \& BW Sobral. 2013. Bacterial DNA sifted from the Trichoplax adhaerens (Animalia: Placozoa) genome project reveals a putative rickettsial endosymbiont. Genome Biology and Evolution 5: 621-645.

Ferris HU, Y Furukawa, T Minamino, MB Kroetz, M Kihara, K Namba \& RM Macnab. 2005. FlhB regulates ordered export of flagellar components via autocleavage mechanism. The Journal of Biological Chemistry 280: 4123641242.

Figueroa-Pizano MD, AB Peregrino-Uriarte, G YepizPlascencia, M Martínez-Porchas, T Gollas-Galván \& LR Martínez-Córdova. 2014. Gene expression responses of white shrimp (Litopenaeus vannamei) infected with necrotizing hepatopancreatitis bacterium. Aquaculture 420: 165-170.

Foynes S, N Dorrell, SJ Ward, ZW Zhang, AA McColm, JG Farthing \& BW Wren. 1999. Functional analysis of the roles of FliQ and FlhB in flagellar expression in Helicobacter pylori. FEMS Microbiology Letters 174: 33-39.

Frelier PF, RF Sis, TA Bell \& DH Lewis. 1992. Microscopic and ultrastructural studies of necrotizing hepatopancreatitis in Pacific white shrimp (Penaeus vannamei) cultured in Texas. Veterinary Pathology 4: 269-277.

Georgiades K, MA Madoui, P Le, C Robert \& D Raoult. 2011. Phylogenomic analysis of Odyssella thessalonicensis forties the common origin of Rickettsiales, Pelagibacter ubique and Reclimonas americana mitochondrion. PLOS ONE 6: e24857. <doi: 10.1371/journal.pone.0024857>

Hyatt D, PF Locascio, LJ Hauser \& EC Uberbacher. 2012. Gene and translation initiation site prediction in metagenomic sequences. Bioinformatics 17: 2223-2230.

Kawabata T \& K Nishikawa. 2000. Protein structure comparison using the markov transition model of evolution. Proteins 41: 108-122.

Krol M, E Hawkins \& M Overstreet. 1991. Rickettsial and mollicute infections in hepatopancreatic cells of cultured Pacific white shrimp (Penaeus vannamei). Journal of the Invertebrate Pathology 57: 362-370.

Levenson R, H Zhou \& FW Dahlquist. 2012. Structural insights into the interaction between the bacterial flagellar motor proteins FliF and FliG. Biochemistry 51: 5052-5060.

Lightner DV, RM Redman \& JR Bonami. 1992. Morphologic evidence for a single bacterial etiology in Texas necrotizing hepatopancreatitis in Penaeus vannamei (Crustacea: Decapoda). Diseases of Aquatic Organism 13: 235-239. 
Loy JK, FE Dewhirst, W Weber, PF Frelier, TL Garbar, SI Tasca \& JW Templeton. 1996. Molecular phylogeny and in situ detection of the etiologic agent of necrotizing hepatopancreatitis in shrimp. Applied and Environmental Microbiology 62: 3439-3445.

Macnab RM. 2003. How bacteria assemble flagella. Annual Review of Microbiology 57:77-100.

Marchler-Bauer A, MK Derbyshire, NR Ganzales, S Lu, F Chitzas, LY Geer, RC Geer, J He, M Gwadz \& DI Hurwitz. 2015. CDD: NCBI's conserved domain database. Nucleic Acids Research 43: 222-226.

Mariconti M, S Epis, L Sacchi, M Biggiogera, D Sassera \& M Genchi. 2012. A study on the presence of flagella in the order Rickettsiales: the case of 'Candidatus Midichloria mitochondrii' . Microbiology 158: 1677-1683.

Martínez-Córdova LR, T Gollas-Galván, E Garibay-Valdez, R Valenzuela-Gutiérrez, M Martínez-Porchas, MA Porchas-Cornejo \& F Mendoza-Cano. 2016. Physiological and immune response of Litopenaeus vannamei undergoing the acute phase of the necrotizing hepatopancreatitis disease and after being treated with oxytetracycline and FF. Latin American Journal of Aquatic Research 44: 535-545.

Minamino T. 2014. Protein export through the bacterial flagellar type III export pathway. Biochimica et Biophysica Acta 1: $1642-1648$

Minamino T \& K Imada. 2015. The bacterial flagellar motor and its structural diversity. Trends in Microbiology-Cell 23: 267-274.

Minamino T \& RM Macnab. 2000. Domain structure of Salmonella FlhB, a flagellar export component responsible for substrate specificity switching. American Society for Microbiology 182: 4906-4914.
Morimoto YV \& T Minamino. 2014. Structure and function of the bi-directional bacterial flagellar motor. Biomolecules 4:217-234.

Morimoto YV, S Nakamura, N Kami-ike, K Namba \& T Minamino. 2010. Charged residues in the cytoplasmic loop of MotA are required for stator assembly into the bacterial flagellar motor. Molecular Microbiology 78: 1117-1129.

Morimoto YV, S Nakamura, KD Hiraoka, K Namba \& T Minamino. 2013. Distinct roles of highly conserved charged residues at the MotA-FliG interface in bacterial flagellar motor rotation. Journal of Bacteriology 195: 474-481.

Nakamura S, YV Morimoto, N Kami-ike, T Minamino \& K Namba. 2009. Role of a conserved prolyl residue (Pro173) of MotA in the mechanochemical reaction cycle of the proton-driven flagellar motor of Salmonella. Journal of Molecular Biology 393: 300-307.

Pallen MJ \& NJ Matzke. 2006. From the origin of species to the origin of bacterial flagella. Nature Reviews Microbiology 4: 784-790.

Sassera D, N Lo, S Epis \& G D'Auria. 2011. Phylogenomic evidence for the presence of a flagellum and cbb3 oxidase in the free-living mitochondrial ancestor. Molecular Biology and Evolution 28: 3285-3296.

Schulz F, J Martijn, F Wascher, I Lagkouvardos, R Konstanjsek, JG Ettema \& M Horn. 2015. A Rickettsiales symbiont of amoebae with ancient features. Environmental Microbiology 1: 1-17. 